\title{
A Novel Glycolysis-related Gene Signature for Survival Prediction in Patients with Head and Neck Squamous Cell Carcinoma
}

\section{Haimei Qin}

Affiliated Hospital of Youjiang Medical University for Nationalities

Junli Wang

Affiliated Hospital of Youjiang Medical University for Nationalities

Biyun Liao

Affiliated Hospital of Youjiang Medical University for Nationalities

Zhonglin Liu

Affiliated Hospital of Youjiang Medical University for Nationalities

Rong Wang ( $\nabla$ rongwang50@163.com)

Affiliated Hospital of Youjiang Medical University for Nationalities

\section{Research Article}

Keywords: Head and neck squamous cell carcinoma, Glycolysis signature, Prognosis, TCGA, GSEA

Posted Date: February 4th, 2021

DOI: https://doi.org/10.21203/rs.3.rs-154414/v1

License: (9) This work is licensed under a Creative Commons Attribution 4.0 International License. Read Full License 


\section{Abstract}

Background: Head and neck squamous cell carcinoma (HNSCC) is most diagnosed at an advanced stage with poor prognosis. Single gene biomarkers cannot have enough predictive ability in HNSCC. Glycolysis participating in cancers was verified. Thus, this study aimed to identify glycolysis-related gene signature predict the outcome of HNSCC.

Methods: The mRNA expression data of HNSCC downloaded The Cancer Genome Atlas (TCGA) project was analyzed by Gene Set Enrichment Analysis (GSEA). We use the Cox proportional regression model to construct a prognostic model. Kaplan-Meier and receiver operating characteristic (ROC) curves were employed to estimate the signature. We also analyzed the relationship of the signature and cancer subtypes.

Results: We identified nine glycolysis-related genes including G6PD, EGFR, ALDH2, GPR87, STC2, PDK3, ELF3, STC1 and GNPDA1 as prognosis-related genes signature in HNSCC. HNSCC patients were divided into high and low risk group according to the signature. High risk group showed more poor prognosis and the risk score can precisely predict the prognosis of HNSCC. Additionally, the signature also can be used in cancer subtypes.

Conclusion: This study established the 9-mRNA glycolysis signature which may serve as a prospective biomarker for prognosis and novel treatment target in HNSCC.

\section{Background}

Head and neck squamous cell carcinoma (HNSCC) includes multiple head and neck cancers and is pervasive cancer that sources from the squamous epithelium of the oral cavity, pharynx and larynx. HNSCC are the sixth in the most common cancers and have approximately 700,000 new patients globally every year [1]. Many HNSCC often attack local part in body with advanced stage and involve lymph nodes. Surgery, radiotherapy and chemotherapy are still the typical treatments. After platinum-based chemotherapy, the one-year survival rate of patients that are not suitable to chemotherapy or keep the progressive situation less than 5\% [2]. Additionally, it often leads to short term and long term morbidity with the fifty percent in cure rate [3]. The most common etiologies of HNSCC comprise smoking and drinking, and human papillomavirus (HPV) infection. A new agent named cetuximab was approved by FDA to therapy this cancer, which has brought hope to patients. But the application of additive cetuximab in chemotherapy only increases 2.7 -month survival and decrease $20 \%$ in the relative risk of death [4]. Therefore, to improve the prognosis for these patients, it is an urgent need to identify more targets.

It is great of importance to identify dependable biomarkers which can predict clinical outcomes of patients for helping us to make decisions in treatment and diagnosis. Recently, some studies have been performed to confirm biomarkers in the prognosis of HNSCC. By using bioinformatics, Guorun Fan et al. [5] found that HSPH1, HSPD1, SERPINH1, HSPA4 and HSP90AA1 genes are potential targets and prognostic biomarkers for head and neck cancer. Patients carrying high expression levels of ALKBH5, 
FTO, METTL14, WTAP, YTHDC1, YTHDF1, and YTHDF2 possessed poor overall survival than patients with low expression [6]. Hao Li et al. [7] suggested that overexpressed IFIT1 and IFITM3 indicated poor prognoses for HNSCC. Non-coding RNA also was observed as biomarkers in HNSCC. Expression levels of miR-let-7a-5p and miR-3928 displayed better sensitivity and specificity in distinguishing HNSCC patients and healthy people [8]. After integrated bioinformatics analysis, Dan Xiong et al. [9] thought that LINC00958 and HOXC13-AS both are new diagnostic biomarkers for HNSCC patients. Nevertheless, the single gene biomarker cannot adequately present good action in prediction. Gene signatures are new means with better effect for predicting prognosis and survival in cancer $[10,11]$.

Emerging findings illustrated that metabolic reprogramming is an important hallmark in the development of cancer [12]. The approach of glycolysis is the most frequent metabolic reprogramming. Aerobic glycolysis named "Warburg effect" has revealed in various cancers. Utilizing this way, glucose is transformed into lactate as a kind of energy. As a result, cancer cells are surrounded by highly acidic microenvironment. it is believed that glycolysis is conducive to the biological behaviors of cancer cells [13]. The new glycolysis-related gene signature has possible implications for predict cancers, such as endometrial adenocarcinoma, diffuse glioma and breast cancer [14-16]. Up to now, the understanding of focusing on the association of glycolysis and the clinical features in HNSCC is limited. Given the significance of tumor glycolysis, we downloaded and analyzed the transcriptase and clinical data of HNSCC from open The Cancer Genome Atlas (TCGA) database. Finally, we look forward to identifying the relationship between glycolysis and HNSCC and finding reliable targets for providing new ideas in treatment of HNSCC.

\section{Methods}

\section{Acquisition of clinical data and mRNA expression}

The Cancer Genome Atlas (TCGA) (https://portal.gdc.cancer.gov/) is a genomics program with clinical features involving 33 cancer types [17]. We directly download the mRNA data of HNSCC with HTseqFPKM extension. A total of 520 tumor samples and 49 normal samples from the database was used for the analysis. The following clinical pathological features: age, gender, stage, survival time and survival status were also download.

\section{Gene set enrichment analysis}

Gene Set Enrichment Analysis (GSEA) can determine two biological states using a set of genes with statistical significance. Molecular Signatures Database (MSigDB) was applied to select the gene sets of glycolysis [18]. GSEA software was installed to analyze the gene sets with the significant differences between the healthy group and the tumor group. $P<0.05$ was the cutoff to choose gene sets for the next investigation. 


\section{cBioPortal database analysis}

cBioPortal (www.cbioportal.org) possessed by the Center for Molecular Oncology at Memorial Sloan Kettering Cancer Center is a public website [19]. Currently, the database is considered a tool to confirm gene mutations because it has been recognized by multiple studies. We used the online cBioPortal to explore mutations of candidate genes in HNSCC.

\section{Statistical analysis}

Expression of all mRNA was calculated by the log2 analysis. We used Cox regression to identify the relationship of genes and overall survival (OS) under the $P$ values less than 0.05 . Consequently, multivariate Cox proportional hazards regression was used to confirm the candidate genes. Hazard ratio was selected to divide the high risk or low risk according which whether surpasses 1 . The risk score module was constructed based on the Cox regression analysis that expression levels times regression coefficients.

\section{Results}

\section{Preliminary analysis of genes through GSEA}

We successfully used the GSEA to analyze glycolysis-related gene sets in the two groups. Five glycolysisrelated gene sets were downloaded and integrated into GSEA. Results demonstrated that the HALLMARK_GLYCOLYSIS gene set with 199 genes (NES=1.99, $P<0.001, F D R<0.001$, Figure 1a, 2a) and REACTOME_GLYCOLYSIS gene set with 71 genes (NES=2.02, $P<0.001$, FDR $<0.001$, Figure 1b, 2b) were significantly enriched with the samples.

\section{Identification of glycolysis-related mRNAs}

Univariate Cox analysis was used to preliminary screening and 43 genes were obtained. Subsequently, multivariate Cox regression analysis evaluated the relation between the expression of 43 genes and the survival of HNSCC. 9 mRNAs including G6PD, EGFR, ALDH2, GPR87, STC2, PDK3, ELF3, STC1 and GNPDA1 were identified as independent indicators of prognosis. Among them, G6PD, EGFR, GPR87, STC2, PDK3, STC1 and GNPDA1 act the role of risk because the HR was > 1. ALDH2 and ELF3 are protective genes with the HR was $<1$, (Table 1$)$. 
Table 1

Information of nine mRNAs significantly associated with survival in HNSCC

\begin{tabular}{|llllll|}
\hline Ensemble ID & Symbol & location & B (cox) & HR & P value \\
\hline ENSG00000160211 & G6PD & X:154531391-154547572 & 0.002107 & 1.002109 & 0.022 \\
\hline ENSG00000146648 & EGFR & $7: 55019017-55211628$ & 0.001515 & 1.001517 & 0.034 \\
\hline ENSG00000111275 & ALDH2 & $12: 111766887-111817532$ & -0.02187 & 0.978367 & 0.005 \\
\hline ENSG00000138271 & GPR87 & $3: 151294086-151316820$ & 0.006771 & 1.006794 & 0.037 \\
\hline ENSG00000113739 & STC2 & $5: 173314713-173329503$ & 0.024477 & 1.024779 & $7.95 \mathrm{E}-6$ \\
\hline ENSG00000067992 & PDK3 & X:24465221-24550466 & 0.071147 & 1.073739 & 0.026 \\
\hline ENSG00000163435 & ELF3 & $1: 202007945-202017183$ & -0.00868 & 0.991355 & 0.060 \\
\hline ENSG00000159167 & STC1 & $8: 23841929-23854806$ & 0.009341 & 1.009385 & $2.33 \mathrm{E}-5$ \\
\hline ENSG00000113552 & GNPDA1 & $5: 141991749-142013041$ & 0.049854 & 1.051117 & 0.023 \\
\hline
\end{tabular}

Using the cBioPortal to assess the alterations in the candidate genes in HNSCC patients. In the patients with HNSCC, they have the 5\% G6PD alteration, 13\% EGFR alteration, 0.4\% ALDH2 alteration, 9\% GPR87 alteration, 06\% STC2 alteration, 2.8\% PDK3 alteration,1.2\% ELF3 alteration, 2.8\% STC1 alteration and $0.6 \%$ GNPDA1 alteration, Figure 3.

\section{Construction of the mRNAs-signature}

A prognostic risk score module was constructed by multivariate Cox regression: Risk score $=0.002107 \times$ expression of G6PD+0.001515xexpression of EGFR-0.02187xexpression of ALDH2+0.006771 xexpression of GPR87+0.024477xexpression of STC2+0.071147xexpression of PDK3$0.00868 \times$ expression of ELF3+0.009341 xexpression of STC1+0.049854×expression of GNPDA1. Based on the risk score equation, we calculated the score of every patient and divided them into the high risk group and the low risk group (Figure $4 \mathrm{a}$ ). As a result, Figure $4 \mathrm{~b}$ shown the mortality rates in patients with the high risk score group was elevated than that in low risk score group. The expression situation of the nine mRNAs was shown in the heatmap (Figure 5). AUC area of the ROC curve was 0.705 which means that the mRNAs signature has good in sensitivity and specificity to predict survival of HNSCC (Figure 6). The increasing risk score of patients have the upregulated G6PD, EGFR, GPR87, STC2, PDK3, STC1 and GNPDA1. The decreasing risk score of patients have the downhearted ALDH2 and ELF3.

\section{Risk score from the nine-mRNA signature is an indicator of prognosis}


Univariate and multivariate analyses were used to analyze clinical pathological parameters for evaluating the value of risk score. In the Figure $7 \mathrm{a}$ and $7 \mathrm{~b}$, we found that the risk score, stage and age were well independent prognostic indicators due to statistical differences in univariate and multivariate analysis $(P<0.05)$. No matter in univariate analysis or multivariate analysis, the risk score represented nice values in prognosis. Similarly, the analysis of survival time and risk score showed that HNSCC patients with the high-risk score exist in the poorer survival (Figure 8). These results further confirmed the accuracy of analysis.

\section{Validation of nine mRNA for expression and survival prediction}

Hence, we continue to analyze the expression of nine mRNA in tumor samples and controls samples. As showed in Figure 9a-9i, the expression of G6PD, EGFR, ALDH2, GPR87, STC2, PDK3, ELF3, STC1 and GNPDA1 was significant differences in tumor samples and control samples $(P<0.01)$. As showed from Figure $10 \mathrm{a}-10 \mathrm{~m}$, regardless of age, gender, $\mathrm{G}, \mathrm{M}, \mathrm{N}, \mathrm{T}$ and stage in tumor, the signature is a stable prognostic marker that patients with the high risk score has more poor prognosis in HNSCC.

\section{Discussion}

Despite advances in improving the roles of genomic and immunity in HNSCC, to date biomarkers is still lacked. Additional biomarkers in the clinical will be needed to guide the prognosis in this cancer. Recently, studies found some mRNAs could be biomarkers to predict the prognosis for HNSCC and indicated they have the clinical significance. For example, Qun Li et al. [20] confirm that levels of P4HA1 mRNA and protein in tissues were obviously higher in HNSCC than that in controls. High expression was related to tumor category, lymphatic metastasis and pathological stage. Xu He et al. [21] downloaded the GSE9716, GSE61772 and GSE20549 from the Gene Expression Omnibus (GEO) database and reported that high POPDC3 expression associated with poor a prognosis for patients with HNSCC. But these genes cannot accurately predict patient survival because a single gene is modulated by many factors. So, gene signature including multiple genes has been the important model to predict outcome in cancers. Results from these signatures reflect more effects and present the more accurate prediction compared to biomarker of a single gene.

Some signatures have been discovered in cancers, a gene glycolysis-related signature consists of HMMR, B4GALT1, SLC16A3, ANGPTL4, EXT1, GPC1, RBCK1, SOD1, and AGRN could be a biomarker which were correlated with metastasis and overall survival in lung adenocarcinoma [22]. Using multivariate analysis, Pankaj Ahluwalia et al. [23] identified a novel prognostic signature which has clinical utility in colorectal cancer. In this study, we also conducted the GSEA to analyze the mRNA expression data from TCGA database and nine mRNAs expression levels have the differences. Additionally, Furthermore, in univariate Cox regression and multivariate Cox regression analyses, we identified that a signature of the nine genes pose the value to predict the prognosis to HNSCC. Consequently, the risk signature also can confirm the 
clinical results. Interestingly, the high-risk score was correlated with a poor prognosis. Glucose-6phosphate dehydrogenase (G6PD) is a vital enzyme in preventing cells from oxidative damage. Some studies have found that G6PD play an important role in cancers to influence its occurrence and development. Chin An Yang et al. [24] suggested that G6PD could be a marker to predict risk, prognosis and chemo-sensitivity of glioma. Epidermal growth factor receptor (EGFR) is a key gene to regulate biological behavior of tumor cells. EGFR-targeted drugs have widely been utilized in therapy of lung and colorectal cancers. Xiaozhen Liu et al. [25] recruited 200 molecular apocrine breast cancer patients and found that EGFR may be helpful to assess the prognosis of the cancer. Expression of ALDH2 were significantly associated with poor prognosis in hepatocellular carcinoma patients [26]. We also found the protective role in prognosis of HNSCC. Intravesical recurrence-free survival of patients with positive GPR87 tumors is shorter than those with negative GPR87 tumors [27]. Overexpression of STC2 can suppress cell apoptosis and promote cell proliferation, migration, invasion, and make cell cycle arresting at the G1/S transition in HNSCC [28]. Consistent with this, in this study, the over expression impacted the poor prognosis of HNSCC. Multivariate analysis indicated that high expression of PDK3 acted an independent risk factor in event-free survival and overall survival of acute myeloid leukemia [29]. ELF3 can regulate the cell cycle and epithelial-mesenchymal transition (EMT) in non-small cell lung cancer through PI3K/AKT and ERK signaling pathways and downstream effectors [30]. H M H Abaza et al. [31] reported expression of STC1 gene could be a useful biomarker for predicting clinical outcome and monitoring therapeutic response in acute leukemia patients. GNPDA1 had good effects on CD4 + memory $T$ cells, which were affected by the gemcitabine therapeutic effect in gemcitabine-resistant pancreatic cancer cell [32]. These results are in line with our that these genes served as carcinogenic roles or cancerocidal roles. As far as know, the mRNAs signature can perform better predict the prognosis of HNSCC.

Increased glycolysis is one of metabolic characteristics named Warburg effect. Deregulation or alteration of energy metabolism has been considered an important hallmark of cancer. Gangcai Zhu et al. [33] revealed that hypoxia can promote migration, invasion and glycolysis in HNSCC by HIF-1 a-metadherin loop. Decreased expression of GRIM-19 enhanced aerobic glycolysis in HNSCC through DNA hypermethylation [34]. Moreover, high expression of HK2 was tested in head and neck cancerous tissues compared to normal counterparts. HK2-deficient HNSCC cells had greater sensitivity to cisplatin and 5fluorouracil [35]. It is crucial that hexokinase is a key enzyme glycolytic prognosis. Hence, metabolism of glycolysis may involve the development and progression of HNSCC. Some single gene involving glycolysis which predict prognosis of HNSCC has been reported in the study, but no report in the glycolysis-related gene signatures. We first reported and illustrated that the glycolysis-gene signature including G6PD, EGFR, ALDH2, GPR87, STC2, PDK3, ELF3, STC1 and GNPDA1 which has prognostic value for HNSCC.

Over all, this study firstly identified that a glycolysis related gene signature (G6PD, EGFR, ALDH2, GPR87, STC2, PDK3, ELF3, STC1 and GNPDA1) can predict the prognosis of patients with HNSCC. This signature could be a new target for the therapy of HNSCC. 


\section{Abbreviations}

HNSCC: Head and neck squamous cell carcinoma; TCGA: The Cancer Genome Atlas; GSEA: Gene Set Enrichment Analysis; ROC: receiver operating characteristic; MSigDB: Molecular Signatures Database; OS: overall survival; GEO: Gene Expression Omnibus; G6PD: Glucose-6-phosphate dehydrogenase; EGFR: Epidermal growth factor receptor; EMT: epithelial-mesenchymal transition.

\section{Declarations}

\section{Acknowledgements}

Not applicable.

\section{Authors' contributions}

Rong Wang designed this study; Haimei Qin wrote the manuscript and performed analysis; Junli Wang and Biyun Liao collected the data; Zhonglin Liu revised the manuscript. All authors read and approved the final manuscript

\section{Funding}

This study was supported by National Natural Science Foundation of China (No. 81560461), Natural Science Foundation of Guangxi province (No. 2019JJB140060), 139 medical high-level talent training plan and thousands of young and middle-aged backbone teacher cultivation plan of Guangxi, high-level talent training plan of Youjiang medical university for nationalities and the affiliated hospital of Youjiang medical university for nationalities.

\section{Availability of data and materials}

The datasets supporting the conclusions of this article are available in the TCGA-GDC (https://portal.gdc.cancer.gov/) repository.

\section{Ethics approval and consent to participate}

Any repository data used in this study are open access and do not require any permissions. Ethics approval and consent to participate are not applicable for them.

\section{Consent for publication}




\section{Competing interests}

The authors declare that they have no competing interests.

\section{References}

1. Bray F, Ferlay J, Soerjomataram I, Siegel RL, Torre LA, Jemal A. Global cancer statistics 2018: GLOBOCAN estimates of incidence and mortality worldwide for 36 cancers in 185 countries. CA: a cancer journal for clinicians. 2018; 68: 394-424.

2. León X, Hitt R, Constenla M, Rocca A, Stupp R, Kovács AF, et al. A retrospective analysis of the outcome of patients with recurrent and/or metastatic squamous cell carcinoma of the head and neck refractory to a platinum-based chemotherapy. Clinical oncology (Royal College of Radiologists (Great Britain)). 2005; 17: 418-24.

3. Pignon JP, le Maître A, Maillard E, Bourhis J. Meta-analysis of chemotherapy in head and neck cancer (MACH-NC): an update on 93 randomised trials and 17,346 patients. Radiotherapy and oncology : journal of the European Society for Therapeutic Radiology and Oncology. 2009; 92: 4-14.

4. Vermorken JB, Mesia R, Rivera F, Remenar E, Kawecki A, Rottey S, et al. Platinum-based chemotherapy plus cetuximab in head and neck cancer. The New England journal of medicine. 2008; 359: 1116-27.

5. Fan G, Tu Y, Wu N, Xiao H. The expression profiles and prognostic values of HSPs family members in Head and neck cancer. Cancer cell international. 2020; 20: 220.

6. Zhou X, Han J, Zhen X, Liu Y, Cui Z, Yue Z, et al. Analysis of Genetic Alteration Signatures and Prognostic Values of m6A Regulatory Genes in Head and Neck Squamous Cell Carcinoma. Frontiers in oncology. 2020; 10: 718.

7. Li H, Yang LL, Wu CC, Xiao Y, Mao L, Chen L, et al. Expression and Prognostic Value of IFIT1 and IFITM3 in Head and Neck Squamous Cell Carcinoma. American journal of clinical pathology. 2020; 153: 618-29.

8. Fadhil RS, Wei MQ, Nikolarakos D, Good D, Nair RG. Salivary microRNA miR-let-7a-5p and miR-3928 could be used as potential diagnostic bio-markers for head and neck squamous cell carcinoma. PloS one. 2020; 15: e0221779.

9. Xiong D, Wu W, Kan L, Chen D, Dou X, Ji X, et al. LINC00958 and HOXC13-AS as key candidate biomarkers in head and neck squamous cell carcinoma by integrated bioinformatics analysis. PeerJ. 2020; 8: e8557.

10. Liang JY, Wang DS, Lin HC, Chen XX, Yang H, Zheng Y, et al. A Novel Ferroptosis-related Gene Signature for Overall Survival Prediction in Patients with Hepatocellular Carcinoma. International journal of biological sciences. 2020; 16: 2430-41. 
11. Wang J, Yu S, Chen G, Kang M, Jin X, Huang Y, et al. A novel prognostic signature of immune-related genes for patients with colorectal cancer. Journal of cellular and molecular medicine. 2020.

12. Hanahan D, Weinberg RA. Hallmarks of cancer: the next generation. Cell. 2011; 144: 646-74.

13. Shao M, Zhang J, Zhang J, Shi H, Zhang Y, Ji R, et al. SALL4 promotes gastric cancer progression via hexokinase II mediated glycolysis. Cancer cell international. 2020; 20: 188.

14. Liu J, Li S, Feng G, Meng H, Nie S, Sun R, et al. Nine glycolysis-related gene signature predicting the survival of patients with endometrial adenocarcinoma. Cancer cell international. 2020; 20: 183.

15. Zhou Z, Huang R, Chai R, Zhou X, Hu Z, Wang W, et al. Identification of an energy metabolism-related signature associated with clinical prognosis in diffuse glioma. Aging. 2018; 10: 3185-209.

16. Li W, Xu M, Li Y, Huang Z, Zhou J, Zhao Q, et al. Comprehensive analysis of the association between tumor glycolysis and immune/inflammation function in breast cancer. Journal of translational medicine. 2020; 18: 92.

17. Goldman MJ, Craft B, Hastie M, Repečka K, McDade F, Kamath A, et al. Visualizing and interpreting cancer genomics data via the Xena platform. Nature biotechnology. 2020; 38: 675-8.

18. Subramanian A, Tamayo P, Mootha VK, Mukherjee S, Ebert BL, Gillette MA, et al. Gene set enrichment analysis: a knowledge-based approach for interpreting genome-wide expression profiles.

Proceedings of the National Academy of Sciences of the United States of America. 2005; 102: 15545-50.

19. Gao J, Aksoy BA, Dogrusoz U, Dresdner G, Gross B, Sumer So, et al. Integrative analysis of complex cancer genomics and clinical profiles using the cBioPortal. Science signaling. 2013; 6: pl1.

20. Li Q, Shen Z, Wu Z, Shen Y, Deng H, Zhou C, et al. High P4HA1 expression is an independent prognostic factor for poor overall survival and recurrent-free survival in head and neck squamous cell carcinoma. Journal of clinical laboratory analysis. 2020; 34: e23107.

21. He X, Xu H, Zhao W, Zhan M, Li Y, Liu H, et al. POPDC3 is a potential biomarker for prognosis and radioresistance in patients with head and neck squamous cell carcinoma. Oncology letters. 2019; 18: 5468-80.

22. Zhang L, Zhang Z, Yu Z. Identification of a novel glycolysis-related gene signature for predicting metastasis and survival in patients with lung adenocarcinoma. Journal of translational medicine. 2019; 17: 423.

23. Ahluwalia P, Mondal AK, Bloomer C, Fulzele S, Jones K, Ananth S, et al. Identification and Clinical Validation of a Novel 4 Gene-Signature with Prognostic Utility in Colorectal Cancer. International journal of molecular sciences. 2019; 20.

24. Yang CA, Huang HY, Lin CL, Chang JG. G6PD as a predictive marker for glioma risk, prognosis and chemosensitivity. Journal of neuro-oncology. 2018; 139: 661-70.

25. Liu X, Feng C, Liu J, Liu J, Li C, Xu C, et al. The importance of EGFR as a biomarker in molecular apocrine breast cancer. Human pathology. 2018; 77: 1-10. 
26. Hou G, Chen L, Liu G, Li L, Yang Y, Yan HX, et al. Aldehyde dehydrogenase-2 (ALDH2) opposes hepatocellular carcinoma progression by regulating AMP-activated protein kinase signaling in mice. Hepatology (Baltimore, Md). 2017; 65: 1628-44.

27. Okazoe H, Zhang X, Liu D, Shibuya S, Ueda N, Sugimoto M, et al. Expression and role of GPR87 in urothelial carcinoma of the bladder. International journal of molecular sciences. 2013; 14: 12367-79.

28. Yang S, Ji Q, Chang B, Wang Y, Zhu Y, Li D, et al. STC2 promotes head and neck squamous cell carcinoma metastasis through modulating the PI3K/AKT/Snail signaling. Oncotarget. 2017; 8: 597691.

29. Cui L, Cheng Z, Liu Y, Dai Y, Pang Y, Jiao Y, et al. Overexpression of PDK2 and PDK3 reflects poor prognosis in acute myeloid leukemia. Cancer gene therapy. 2020; 27: 15-21.

30. Wang H, Yu Z, Huo S, Chen Z, Ou Z, Mai J, et al. Overexpression of ELF3 facilitates cell growth and metastasis through PI3K/Akt and ERK signaling pathways in non-small cell lung cancer. The international journal of biochemistry \& cell biology. 2018; 94: 98-106.

31. Abaza HM, Elmougy MI, El Maraghy HM, Mahmoud HM. Stanniocalcin1 gene expression in patients with acute leukemia: impact on response to therapy and disease outcome. International journal of laboratory hematology. 2016; 38: 81-9.

32. Gu J, Zhang J, Huang W, Tao T, Huang Y, Yang L, et al. Activating miRNA-mRNA network in gemcitabine-resistant pancreatic cancer cell associates with alteration of memory CD4(+) T cells. Annals of translational medicine. 2020; 8: 279.

33. Zhu G, Peng F, Gong W, She L, Wei M, Tan H, et al. Hypoxia promotes migration/invasion and glycolysis in head and neck squamous cell carcinoma via an HIF-1a-MTDH loop. Oncology reports. 2017; 38: 2893-900.

34. Zhang XY, Li M, Sun K, Chen XJ, Meng J, Wu L, et al. Decreased expression of GRIM-19 by DNA hypermethylation promotes aerobic glycolysis and cell proliferation in head and neck squamous cell carcinoma. Oncotarget. 2015; 6: 101-15.

35. Li WC, Huang CH, Hsieh YT, Chen TY, Cheng LH, Chen CY, et al. Regulatory Role of Hexokinase 2 in Modulating Head and Neck Tumorigenesis. Frontiers in oncology. 2020; 10: 176.

\section{Figures}



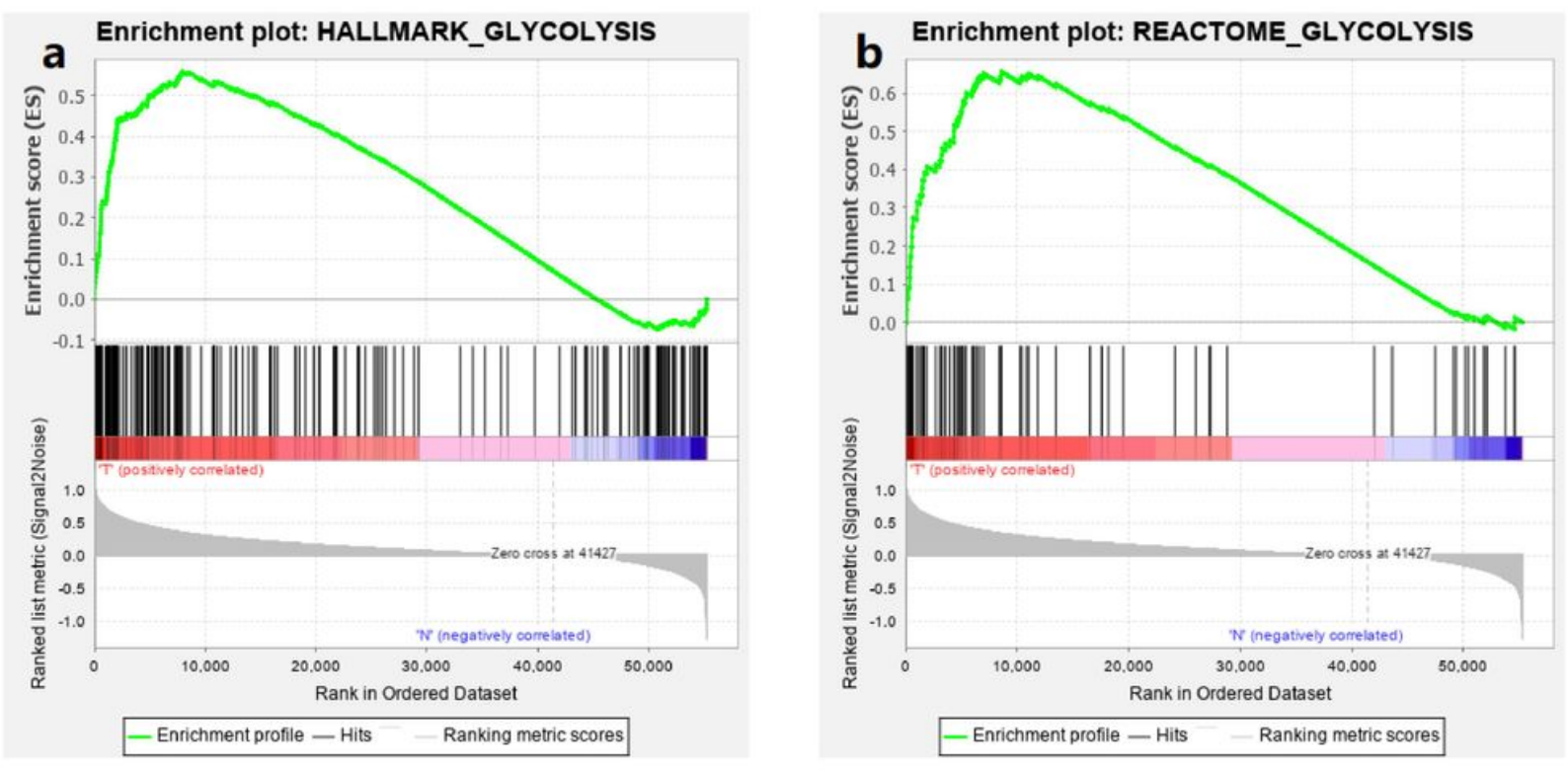

Figure 1

\section{Figure 1}

GSEA from glycolysis-related gene sets. a. Enrichment graph of HALLMARK_GLYCOLYSIS gene set between cancer group and the normal group. b. Enrichment graph of REACTOME_GLYCOLYSIS gene set between cancer group and a normal group. 

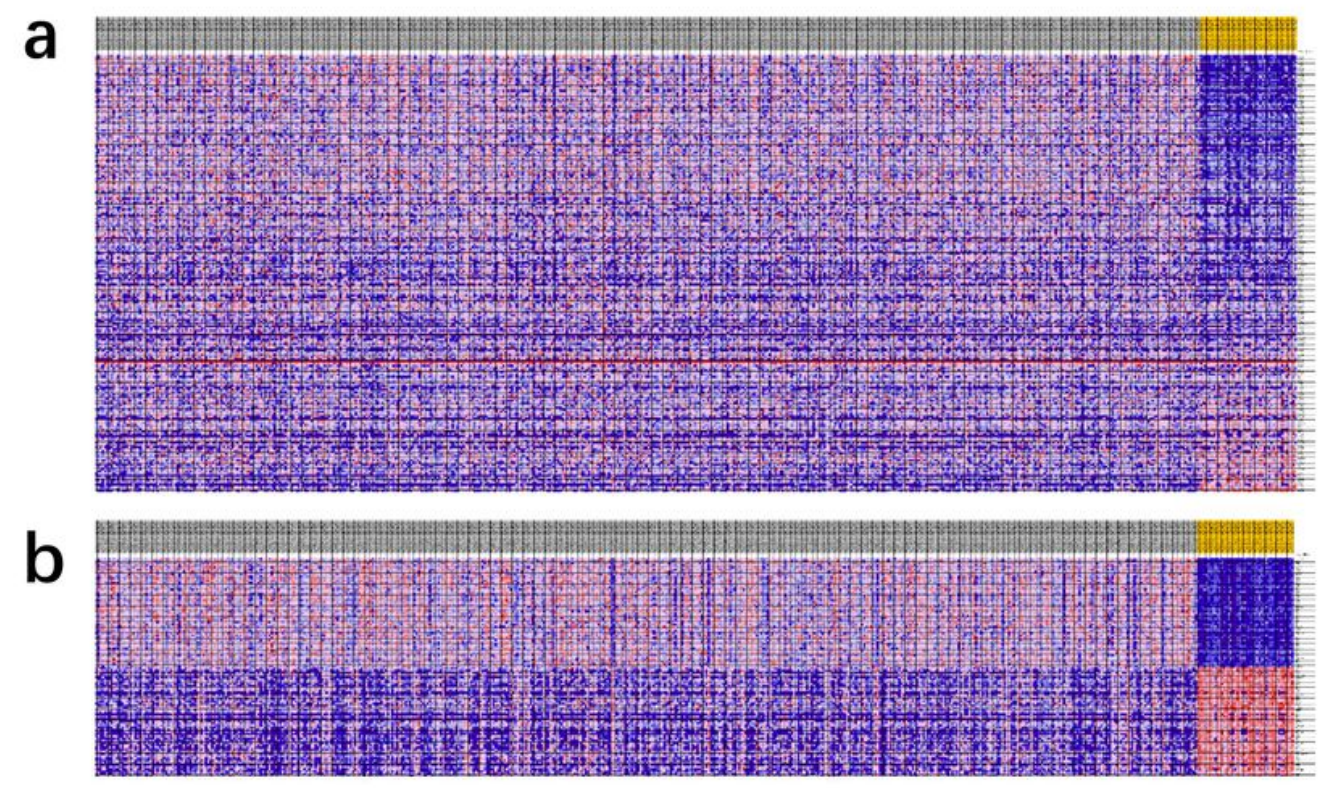

Figure 2

\section{Figure 2}

Selected genes' sets. a. HALLMARK_GLYCOLYSIS gene set b. REACTOME_GLYCOLYSIS gene set

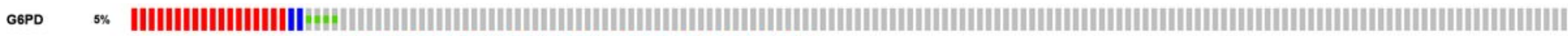

EgF

AL

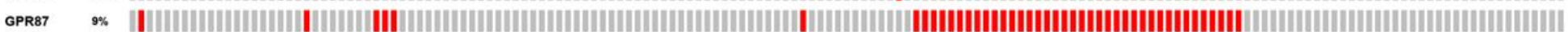

sтc2 0.056 |

рокз $28 \%$.

Eะร3

sтc1 2 28s

GNoA9

Genetic Alteration

it Missense Mutation (putative driver) I| Missense Mutation (unknown significance)

if Truncating Mutation (unkno

| Deep Deletion II No a alterations

\section{Figure 3}

The alterations in the candidate genes by cBioPortal in HNSCC patients. Construction of the mRNAssignature 

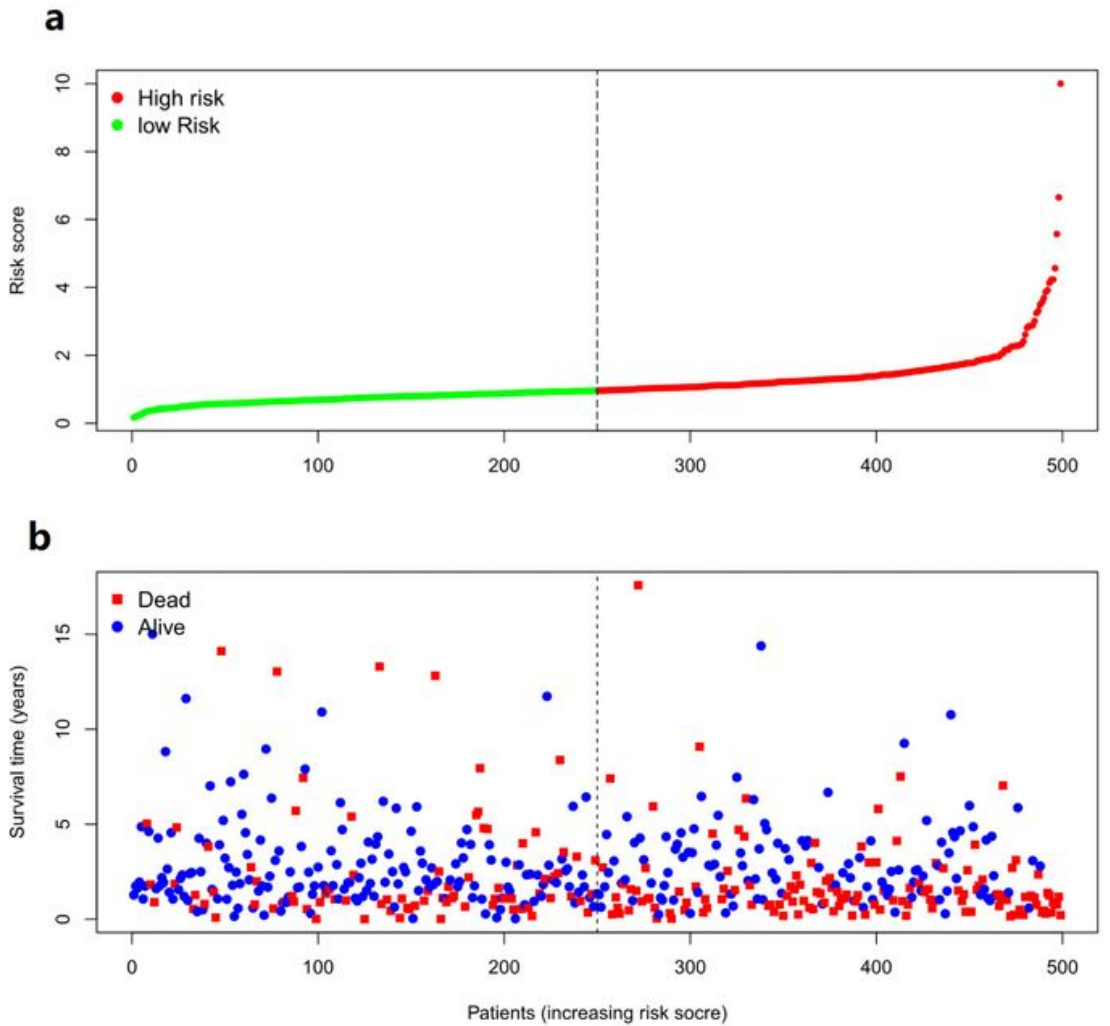

figure 4

Figure 4

The risk score predicts survival status in the patients of HNSCC.

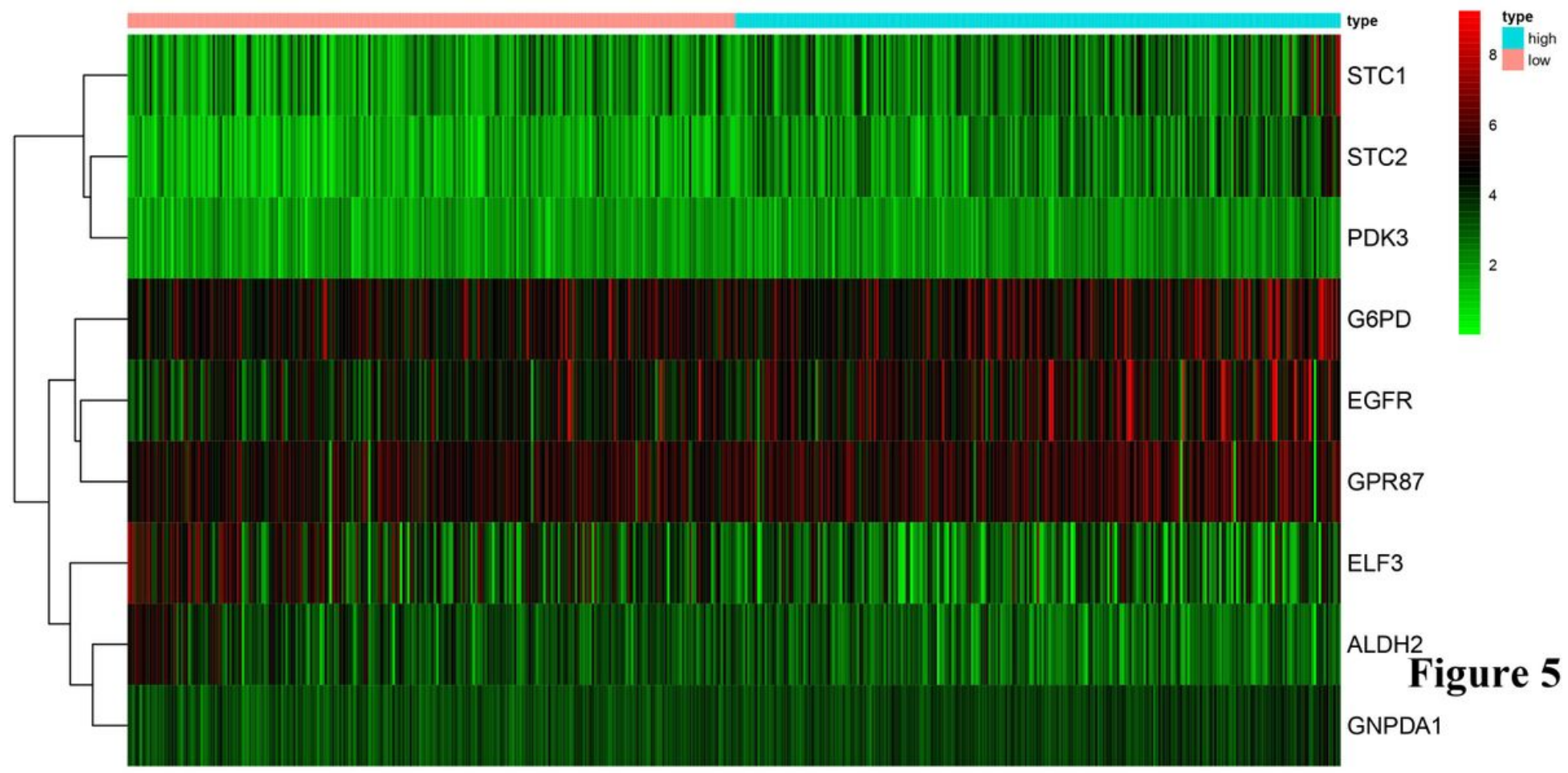

Figure 5 
Heatmap of nine genes' mRNA expression profile.

ROC curve ( AUC $=0.705$ )

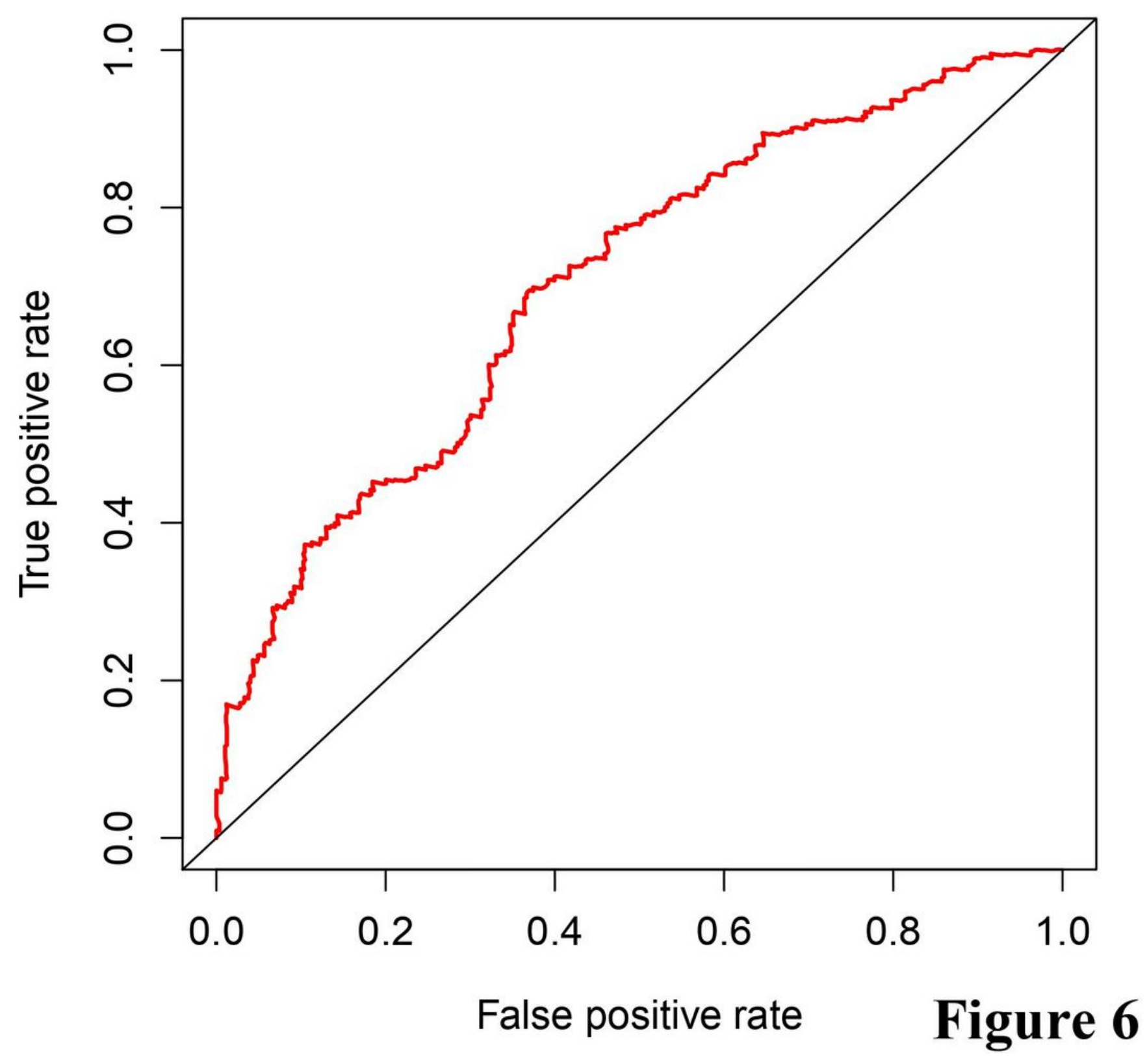

Figure 6

Receiver operating characteristic (ROC) of the risk score model. 


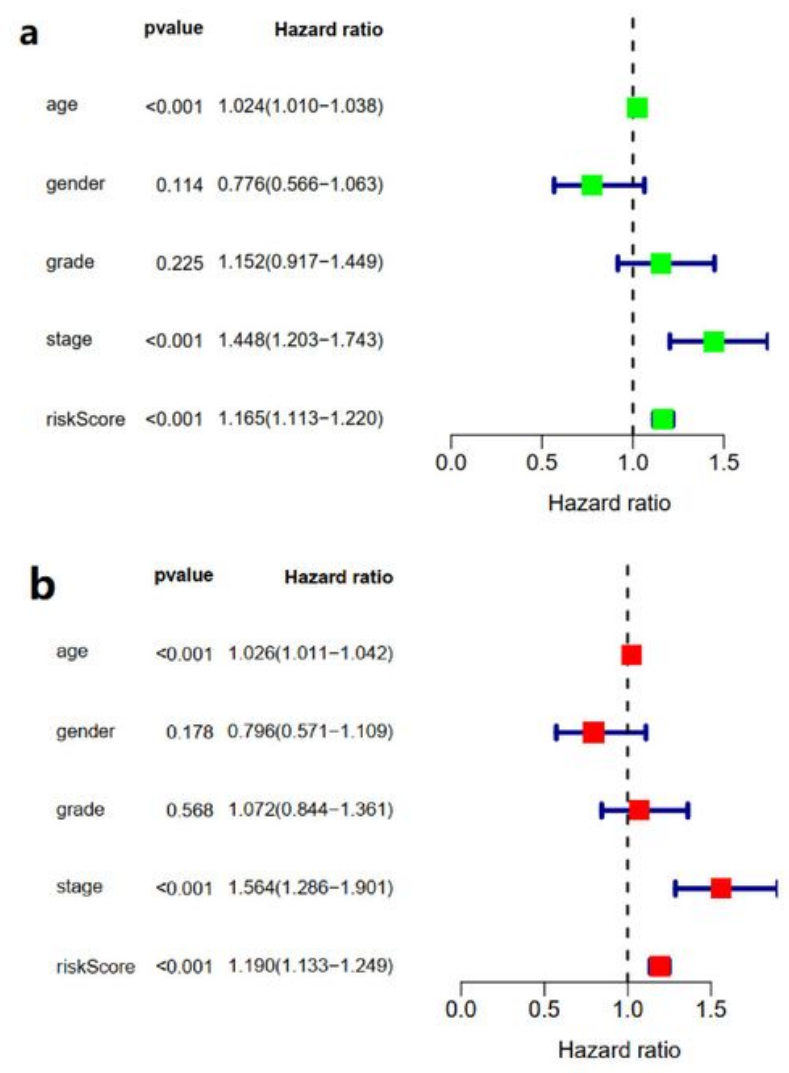

Figure 7

\section{Figure 7}

Univariable analyses and multivariable analysis of the risk score and clinical features. a. Univariable analysis b. multivariable analysis 
Risk $+\operatorname{high}(n=249)+\operatorname{low}(n=250)$

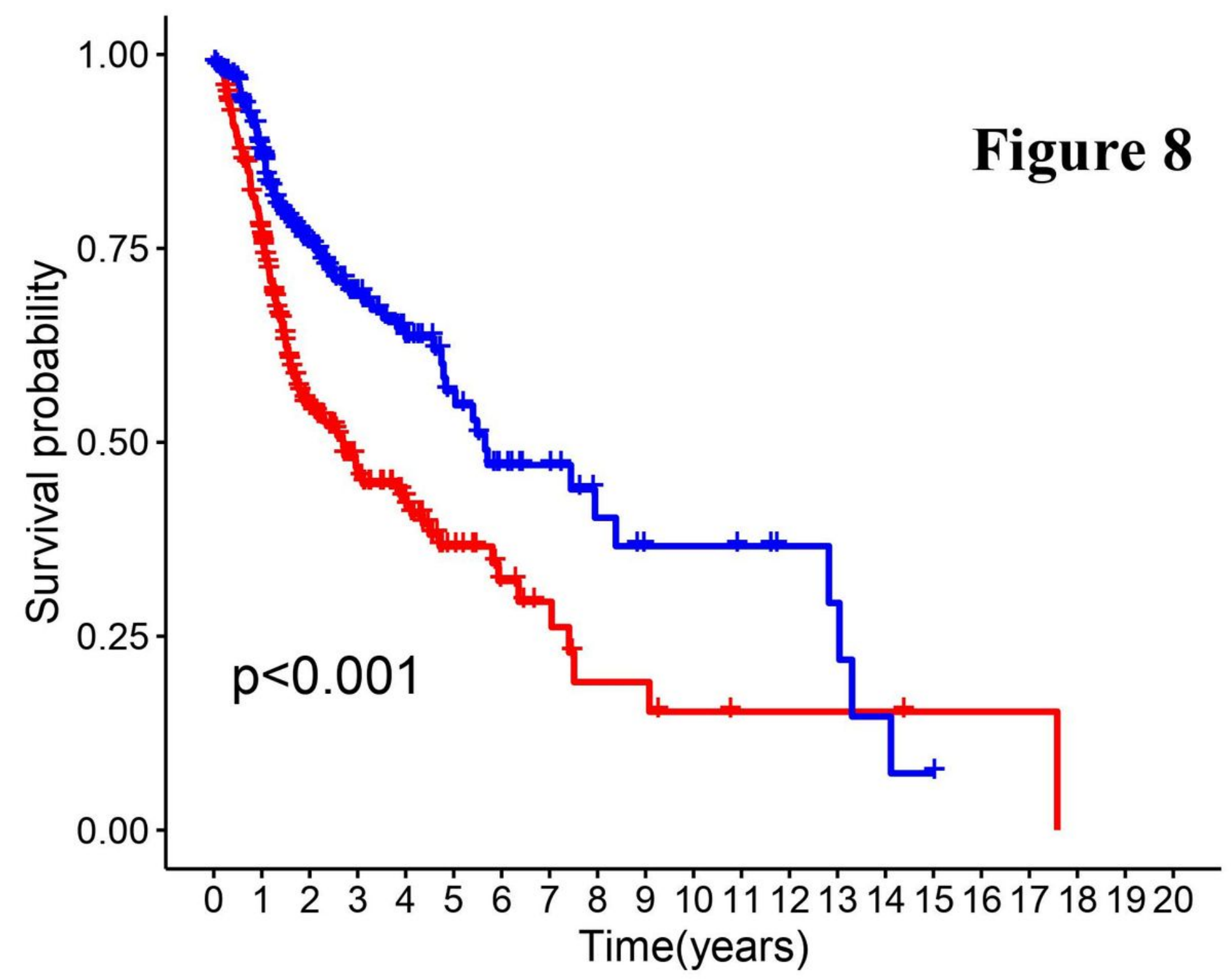

Figure 8

Kaplan-Meier curve of the survival at high- and low-risk groups. 

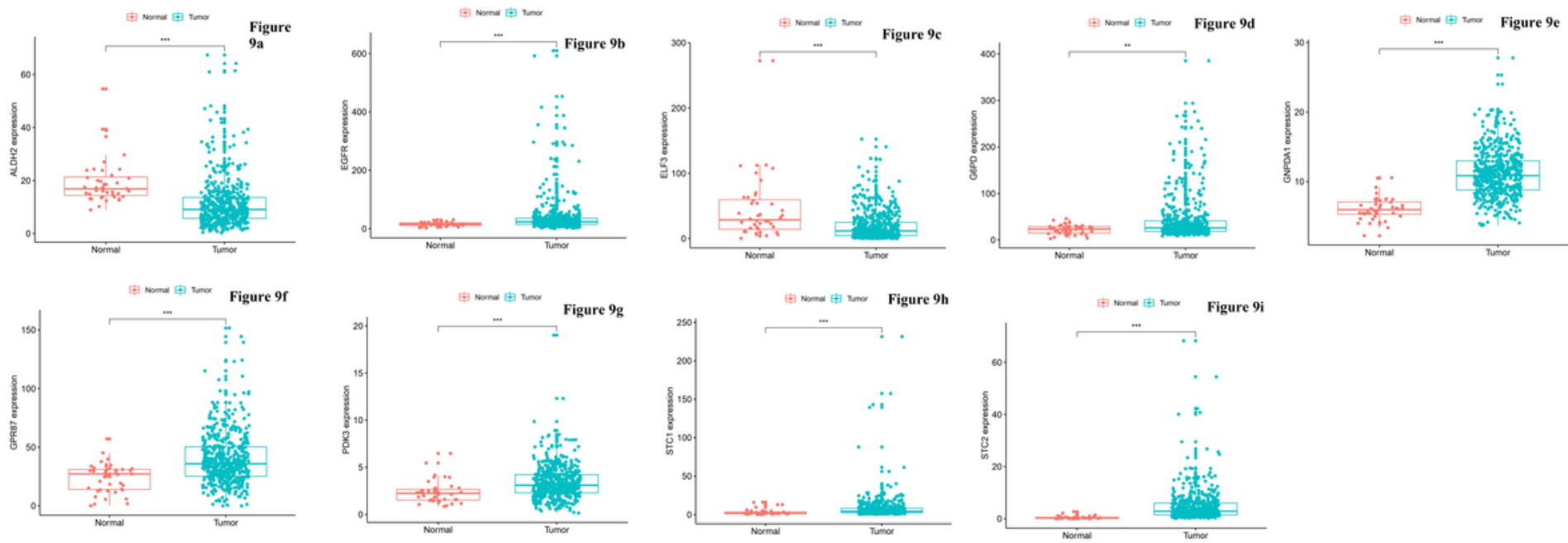

Figure 9

The expression of nine mRNA in tumor samples and controls samples. $* \star \star, P<0.001 ; * \star, P<0.01$
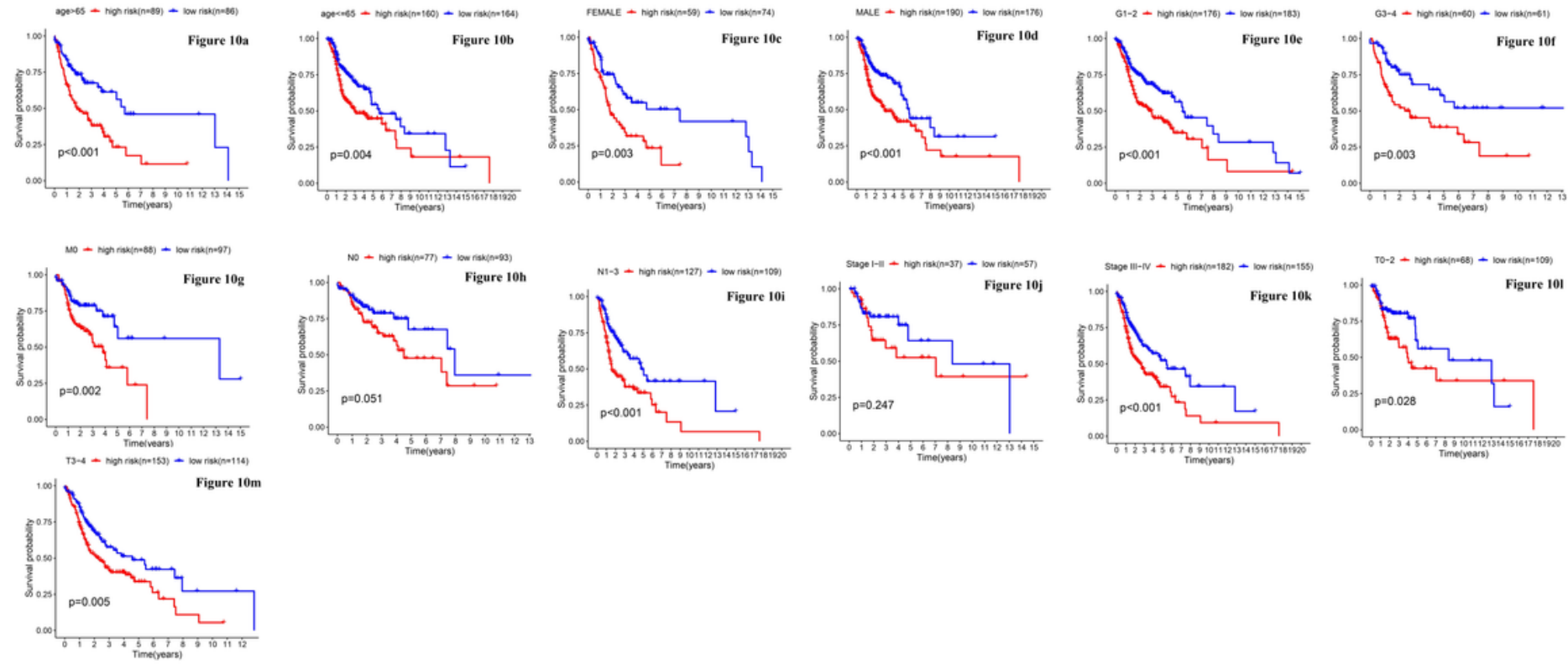

Figure 10

Different clinical features predict the survival of patients with HNSCC. Discussion 